\section{A REVIEW OF RELAY NETWORK ON UAVS FOR ENHANCED CONNECTIVITY}

\author{
Muhammad Nauman Bashir, Kamaludin Mohamad Yusof*
}

School of Electrical Engineering, Faculty of Engineering, Universiti Teknologi Malaysia, 81310 UTM Johor Bahru, Johor Malaysia
Article history

Received

20 August 2019

Received in revised form

26 November 2019

Accepted

8 December 2019

Published online

25 December 2019

*Corresponding author kamalmy@utm.my.

\begin{abstract}
One of the best evolution in technology breakthroughs is the Unmanned Aerial Vehicle (UAV). This aerial system is able to perform the mission in an agile environment and can reach the hard areas to perform the tasks autonomously. UAVs can be used in post-disaster situations to estimate damages, to monitor and to respond to the victims. The Ground Control Station can also provide emergency messages and ad-hoc communication to the Mobile Users of the disaster-stricken community using this network. A wireless network can also extend its communication range using UAV as a relay. Major requirements from such networks are robustness, scalability, energy efficiency and reliability. In general, UAVs are easy to deploy, have Line of Sight options and are flexible in nature. However, their 3D mobility, energy constraints, and deployment environment introduce many challenges. This paper provides a discussion of basic UAV based multi-hop relay network architecture and analyses their benefits, applications, and tradeoffs. Key design considerations and challenges are investigated finding fundamental issues and potential research directions to exploit them. Finally, analytical tools and frameworks for performance optimizations are presented.
\end{abstract}

Keywords: Unmanned Aerial Vehicle, relay network, optimization, network coverage expansion

(C) 2020 Penerbit UTM Press. All rights reserved

\subsection{INTRODUCTION}

The unique features of Unmanned Aerial Vehicles (UAV) or drones, enable them to be utilized in a wide range of applications and it is expected their number will skyrocket in a few years. Historically they have been used in military and mission-critical application but now they are finding use in civilian and commercial applications as well. Since UAVs can easily move in the air and can carry communicating equipment, they are being utilized in mapping and localization, rescue and search operations [1], fire detection, surveillance, police operations, building and engineering inspections, aerial photography, video for post-disaster assessments, agricultural monitoring, remote detection, weather services, commercial, recreational, and educational domains [2]-[3]. Apart from other applications of UAVs as a relay, one promising use is in high-speed broadband wireless communication ad-hoc network in areas where there is no infrastructure. Most of the current research work carried out related to UAVs is focused on issues like navigational control, dynamics, autonomous operation, etc. while its study of wireless communication usage and associated challenges are recent[4] topics.

In terrestrial cellular wireless networks, Ground Control Station (GCS) or Ground Base Stations (GBS) are statically positioned, are typically two dimensional (2D) for static cell association operations and provide long-term and permanent communication without timing and energy constraints. However, GBS of terrestrial wireless networks cannot maintain Line of Sight (LOS) communication in certain situations. UAVs on the other hand, if used as Relay Stations (RS) or Aerial Relay Station between GBS and End Users (EUs) 
have the ability to extend wireless network connectivity [5]. These flying machines can efficiently extend the connectivity and capacity of terrestrial cellular networks or broadband networks establishing LOS communication as relays. A good example is Facebook Aquila, high altitude solar-powered drone system to provide internet access to remote areas [6].

Attractive features of UAV, that enable its use in difficult areas without infrastructure are that they can easily hover, have flexible cell association capabilities, can provide three dimensional (3D) mobility and flexible coverage, can provide on-demand short-term communication, can support frequency variations, can maintain LOS communication links and can be deployed easily compared to GBSs as relay. In a range of applications, single UAV becomes inefficient to meet the mission requirements which can be supported with multiple UAVs deployments. The UAVs in cooperative network topology, acting as EUs as well as RS can deliver reliable and on-demand data. UAVS can also facilitate communication range of ground wireless devices in border surveillance applications. The Internet of Things (IOT) applications where devices have limitations on power, frequency and range can also be supported by UAVs. Figure 1 shows a scenario where UAVs as relay nodes are being controlled by a GBS to monitor an area of interest in tree topology.

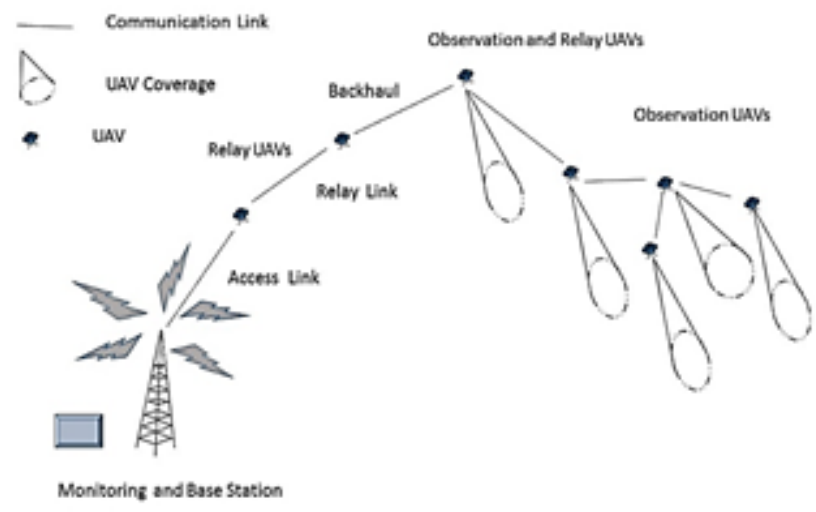

Figure 1 UAVs Aided Wireless Communication

Classification of UAVs based on their type of flight could be as rotary wings or fixed wings. Other options are balloons and airships. Fixed-wing UAVs are heavy, have high speed, have long flight duration, can move in forwarding direction only, are used at high altitudes only, cannot hover and can carry light payloads like aircraft. While rotary-wing UAVs can hover, can remain stationary, fly at low altitudes, are flexible, are limited with energy and are for short duration flights only like quadrotor drones [7]. Fixed-wing UAVs are more suitable towards critical missions while rotarywing UAVs due to their flexibility are more suitable for the communicational environment. A rotary UAV having good control over its altitude can establish and maintain communication links with GBS and or other UAVs. Quality of Service (QoS), characteristics of the area of deployment and national/international regulations define the selection of UAVs for particular communication requirements [8].

The quadrotor UAVs have strict energy constraints, do lack efficient energy models and have flight time limitations. Similarly, UAVs assisted multi-hop relay networks, face many other technical challenges like UAV deployment, efficient channel models, management of handover process, control of latency, characterization of performance, allocation of resources, optimization of trajectory control and efficient planning of network [4] which need efficient solutions.

This paper provides an overview of UAVs assisted networking by reviewing important literature that discusses issues and opportunities in using UAVs as relay nodes. Section 2 provides a review of applications and examples of UAVs in wireless networking. Section 3 provides the main research challenges and possible solutions. Section 4 provides a roadmap for further research in identified directions. Section 5 concluding remarks on this article.

\subsection{WIRELESS NETWORKING WITH UAVs}

UAVs as wireless communication relay nodes find applications in various scenarios like coverage areas expansion, public safety, hard area coverage, and loT support. This section discusses a few of such examples.

\subsection{Network Capacity and Coverage Enhancement Strategies UAVs as Relays}

In communication systems, users should get quality of experience (QoE) in service without delay, without error, and without signal loss. Point to multi-point (PMP) architecture consisting of cellular GBSs and mobile stations (MS) having only direct links are common architecture for 3G where the GBSs are expected to serve multiple MSs in coverage area simultaneously. But in situations when MS is not getting a proper signal due to the shadow of a building or tree (shadowing effect or coverage hole) or if it is at far distance from GBS, it will receive a weak signal and hence low data rate. The wireless networks are also limited by the RF spectrum, coverage, geography and by the presence of time-varying channels due to the mobility of users.

To enhance the coverage and number of connections, ad-hoc solutions like the multi-hop mesh network of fixed antennas or mobile antennas like UAVs based on mesh topology working with terrestrial cellular networks may be utilized as illustrated in Figure 2. In mesh networks routing is carried out by the user equipment and requires efficient frequency planning and efficient resource allocation which is more challenging if the cellular network is dense and if the cell size is of smaller category [9]. In hard geographical areas, deployment of a mesh of fixed antennas or UAVs is even more challenging due to the challenges 
like interference, back-haul, blockage, and failure in maintaining promised high speed and low latency.

An efficient solution to meet the above-mentioned issues is the use of fixed antennas or mobile antennas like UAVs as multi-hop relays based on tree topology working with the main terrestrial cellular network. In relay architecture along with GBSs and MSs, RSs are installed to provide the signal to MSs in coverage holes or in non-coverage areas. These relays are working at LOS with the main GBS. Compared to the concept of repeaters, RSs have some GBS functionalities and, have moderate cost and can be deployed fast.

Since received power determines the data rate for the user and path loss is difficult to mitigate, hence for higher data rates, transmit power should be raised, however. But it also increases the interferences. One good feature which leads to their selection as relays in cellular wireless networking is their reduced transmit power being at a lesser distance to MSs and less cost compared to GBS. RS-based cellular networks need less power to be transmitted to maintain a high data rate. Secondly, separate backhaul is not required for RSs as they can utilize the existing GBS backhaul in urban areas where installation costs are high and even most of the time, options are not available. The RSs can be installed and removed easily to address coverage holes of cellular systems increasing their coverage and capacity as described by WiMAX (IEEE $802.16 \mathrm{j}$ and IEEE $802.16 \mathrm{~m}$ ) and LTE-A [10] standards. In emerging cellular networks, the RSs are aimed to increase the coverage density to one million MSs in a square kilometer area. Relay architecture can also serve MSs in buses, trains, shadowed regions and areas close to cells where signal are not present.

Relay transmission modes are either transport or non-transport type. Mainly, the transport mode is to maximize capacity and to handle shadowing but cannot work independently since scheduling is performed by GBS. On the other hand, the nontransport mode is to maximize coverage as RS is equipped with some of the functionalities of GBS like generating control signals, monitoring and call metering, etc. Relays are working either as Amplify and Forward (AF) or Decode and Forward (DF). In AF mode, the signal is just amplified and transmitted without processing however noise is also amplified and transmitted. In DF mode, relay demodulates signal checking for the threshold to generate a fresh signal and then modulates again using FEC [11]. However, the quality of transmission is achieved at the cost of introduced delay. Efficient algorithms are required to handle these issues. Relays can also be divided into three categories based on their mobility. Mobile Relay Stations (MRS) are for public transport as buses running at 50 or $70 \mathrm{kmph}$ which need fast switching among various cells. Fixed Relay Stations (FRS) for fixed applications or locations as event coverage or capacity enhancement or coverage enhancement or quality enhancement. Nomadic Relay Stations (NRS) are for slow-moving users.

A comparison of RS with various types of cellular GBS is given in Table 1. This table compares needed transmit powers, coverage distance, bandwidth, user capacity, and installation costs.

Table 1 Comparison of RSs with various types of GBS

\begin{tabular}{|l|l|l|l|l|}
\hline & $\begin{array}{l}\text { Macro } \\
\text { GBS }\end{array}$ & Micro GBS & Pico GBS & RS \\
\hline $\begin{array}{l}\text { Transmit } \\
\text { Power }\end{array}$ & $20 \mathrm{~W}-40 \mathrm{~W}$ & $2 \mathrm{~W}-20 \mathrm{~W}$ & $\begin{array}{l}0.25 \mathrm{~W}- \\
2 \mathrm{~W}\end{array}$ & $2 \mathrm{~W}-20 \mathrm{~W}$ \\
\hline Coverage & $3 \mathrm{~km}$ & $\begin{array}{l}0.25 \mathrm{~km}- \\
1 \mathrm{~km}\end{array}$ & $\begin{array}{l}0.1 \mathrm{~km}- \\
0.3 \mathrm{~km}\end{array}$ & $\begin{array}{l}1 \mathrm{~km} \\
1.25 \mathrm{~km}\end{array}$ \\
\hline Bandwidth & $100 \mathrm{MHz}$ & $100 \mathrm{MHz}$ & $30 \mathrm{MHz}$ & $50 \mathrm{MHz}$ \\
\hline $\begin{array}{l}\text { User } \\
\text { Capacity }\end{array}$ & $\begin{array}{l}\text { GMicro } \\
\text { GBS }\end{array}$ & $>$ Pico GBS & 32 & $\begin{array}{l}>\text { Pico } \\
\text { GBS }\end{array}$ \\
\hline $\begin{array}{l}\text { Cost } \\
\text { (2014) }\end{array}$ & $\begin{array}{l}\text { O.4 Million } \\
\text { Euros }\end{array}$ & $\begin{array}{l}42 \\
\text { Thousand } \\
\text { Euros }\end{array}$ & $\begin{array}{l}12.4 \\
\text { Thousand } \\
\text { Euros }\end{array}$ & $\begin{array}{l}56 \\
\text { Thousand } \\
\text { Euros }\end{array}$ \\
\hline
\end{tabular}

\subsection{UAVs as Relay Nodes in Various Communication Scenarios}

UAVs are fast, flexible and dynamic in nature, and if used as relay nodes with a wireless communication network, they can extend the range of communication and can enhance the capacity of the network disseminating on-demand communication at low cost.

Table 2 Relay Applications in Area and Network Coverage

\begin{tabular}{|c|c|c|c|}
\hline & $\begin{array}{c}\text { Small Sized } \\
\text { Area }\end{array}$ & $\begin{array}{c}\text { Medium } \\
\text { Sized Area }\end{array}$ & $\begin{array}{c}\text { Large Size } \\
\text { Area }\end{array}$ \\
\hline $\begin{array}{c}\text { Area } \\
\text { Coverage }\end{array}$ & $\begin{array}{l}\text { - Structure } \\
\text { and surface } \\
\text { monitoring } \\
\text { - construction } \\
\text { site } \\
\text { monitoring } \\
\text { - mapping of } \\
\text { archaeologi } \\
\text { cal sites } \\
\text { - bridge } \\
\text { inspection }\end{array}$ & $\begin{array}{l}\text { - Vegetation } \\
\text { monitoring } \\
\text { - aerial } \\
\text { photography } \\
\text { - event } \\
\text { coverage } \\
\text { and } \\
\text { journalism } \\
\text { - mapping of } \\
\text { disasters } \\
\text { - surveys } \\
\text { - VANET. }\end{array}$ & $\begin{array}{l}\text { - Wildfire } \\
\text { monitoring } \\
\text { - highway } \\
\text { surveillance } \\
\text { - maritime } \\
\text { surveillance } \\
\text { - border } \\
\text { surveillance } \\
\text { - disaster } \\
\text { mapping }\end{array}$ \\
\hline $\begin{array}{c}\text { Network } \\
\text { Coverage }\end{array}$ & $\begin{array}{l}\text { - Network } \\
\text { coverage in } \\
\text { obstacle } \\
\text { ridden } \\
\text { mapping } \\
\text { - Communica } \\
\text { tion relay in } \\
\text { underwater } \\
\text { vehicles. }\end{array}$ & $\begin{array}{l}\text { - Temporary } \\
\text { network } \\
\text { coverage for } \\
\text { mobile users } \\
\text { of sport } \\
\text { events } \\
\text { - ground WSN } \\
\text { coverage } \\
\text { - enhancing } \\
\text { network } \\
\text { throughput } \\
\text { of MANET } \\
\text { - maintaining } \\
\text { connectivity, }\end{array}$ & $\begin{array}{l}\text { - loT data } \\
\text { collection } \\
\text { - Emergency } \\
\text { network } \\
\text { coverage in } \\
\text { disaster } \\
\text { situations } \\
\text { - public safely } \\
\text { - target } \\
\text { tracking } \\
\text { - WSN data } \\
\text { collection }\end{array}$ \\
\hline
\end{tabular}


Few Application Scenarios are:

\section{UAVs to Support Cellular GBS as Relays}

Increase in the growth of UAVs in various applications has provided a direction to utilize them for wireless access as relays especially in $5 \mathrm{G}$ systems that have advanced requirements. Flexibility and control over the height of UAVs make them an ideal option to enhance ground communication in terms of coverage and QoE. A series of such relays can forward EU data to the base station in a multi-hop fashion. These mobile UAV relays can solve coverage issues as well can provide services to the hotspots as illustrated in Figure 2. Authors in [12] have proposed a multiple access mechanism based on distance-time duplex for UAV-GBS link along with Bayesian statistical inference framework using localization algorithm. Similarly, UAVs can also support terrestrial cellular networks to spread information over larger areas as connectivity enhancer. Such UAVs can also share the traffic load of cellular network improving network capacity. Authors in [7] elaborate the UAVs deployment as relay nodes in ad-hoc networks to support GBS to extend communication range and discuss related positioning and physical layer challenges due to UAVs agility and mobility nature. A survey in [13] discusses channel models of UAVs and their networking issues.

\section{UAVs for Public Safety and Post Disaster Estimations}

Terrestrial wireless networks have been affected by natural disasters in recent years. For example, the East Japan earthquake damaged the communication infrastructure resulting in isolation, making it almost impossible to access the disaster-affected area resulting in delayed rescue efforts. Other natural disasters as flood, storms and tornados can also bring large scale damages as illustrated in Figure 2. In such critical situations, the available cellular base stations can be overloaded and need alternate fast and capable links using UAV relays at least during safety operations and during damage estimations [14] forming a flying ad-hoc network (FANET) of UAVs.

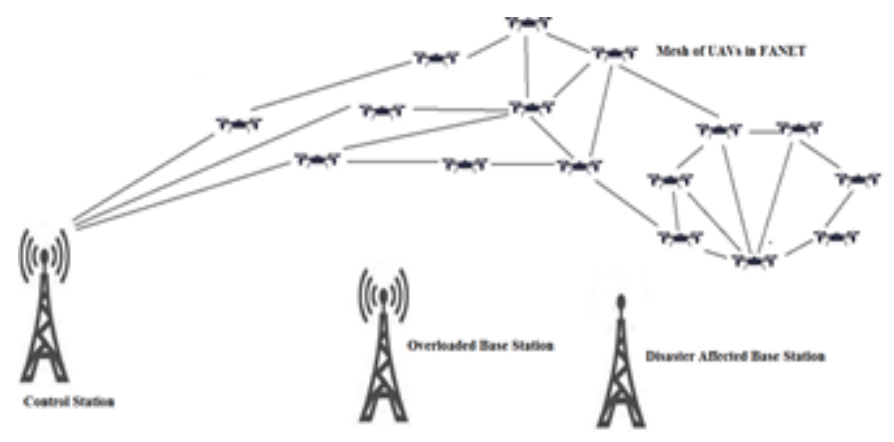

Figure 2 UAVs Aided Cellular Wireless Communication in Disaster Affected Area
A good feature of UAVs, which are flying base stations to establish an ad-hoc network is that they can hover to provide the desired coverage so that UAV assisted cellular network can spread the emergency messages in public safety situations. In disaster situations low latency, reliable and flexible UAVs deployment can be used as they do not require huge infrastructure and can easily establish an on-demand safety communication network.

\section{UAVs for Surveillance Applications}

For surveillance applications, uplink data requirement is more which is usually not supported by cellular networks. UAVs as relays to the existing cellular networks are efficient solutions in such scenarios. However such arrangements have different channel conditions, which are limited by onboard energy constraints and channel variations due to the mobility dynamics of UAVs which need better design considerations to better work with terrestrial cellular networks. A framework is presented in [15] to monitor Smart city using UAVs in collaboration with existing transportation means and wide ocean area using UAVs at various layers through airborne docking.

\section{UAVs for On-demand Communication}

Hard geographical areas without network coverage and occasional events may also be served by ondemand network access temporarily using UAVs. Few organizations are even now using such arrangements to cover events like football championships. Due to the unique features of UAVs, they can be an attractive solution for reliable Device to Device (D2D) and Vehicle to Vehicle (V2V) communications as well. If the UAVs do carry MIMO capabilities, they can be an attractive solution for high capacity, high-speed data provision as they can transfer information to GBS and other UAVs rapidly [16]. UAVs can also support vehicular networks by spreading useful information to all connected vehicles improving reliability. One option may be a grouping or clustering the users and providing services through UAVs where each UAV is communicating to the server of the group, performing improved multi-hop communication.

\section{Demand Aware Backhaul Allocation using UAV Relays}

In hierarchical wired communication networks, the portion of the network consisting of few inter-links among backbone and subnet is termed as backhaul which may be affected by the geographical nature of the area of service. Wireless backhaul is a costeffective solution in some cases, however, interference and blockage are major challenges [17] which may be handled by using UAV relays. $\mathrm{mmW}$ capabilities and reconfigurable capabilities with UAV relays can also provide high quality, reliability, and capacity. 


\section{QoE Enhancement using UAVs}

UAVs due to their unique features of deployment can be considered as flying antennas in 2D MIMO, Full MIMO, and $\mathrm{mmW}$ applications. Support to a large number of users, reduction in inter-cell interference, enhancement in network throughput can be achieved using efficient 3D beamforming [18]. If these airborne base stations are present at high altitudes, they can form good communication links at different altitudes and angels. UAV based antenna arrays have flexibility on space constraints of antenna elements, on beamforming gains, and on beam-steering which may lead to massive MIMO deployment to achieve $\mathrm{mmW}$ communication. Similarly if Swarm of UAVs is deployed, it may simulate reconfigurable antenna arrays.

\section{Cache Enabled UAVs for QoS}

In small dense cells, caching is a good method to have better throughput and reduced latency however it is affected by the handover process [19]. $U A V$ relays as reduced functionality base stations can be a potential solution in such cases that can track user mobility and data and can cache necessary data and mobility pattern to pass-on it to the neighboring cells during the hand over process. If UAVs are connected to cellular base stations as EUs they can be used for delivery, sensing, and surveillance as well.

\section{UAVs for loT Applications}

IOT applications like smart cities, healthcare, energy management and transportation need quality communication among massive IOT devices with a high data rate, reliable and low latency data communication. Since IOT devices are limited to transmission coverage and energy and cannot deliver data to far base stations effectively, UAV relays can be an effective solution especially in scenarios where there is no cellular coverage [20]. In such deployments, IOT devices need to transmit low power to uplink information to UAV relays. UAVs can also support smart cities' requirements as missions, radio map sensing, big data collection, limited processing and efficient transportation of data to the central cloud.

Numerous application can be supported by UAVs, but there are many technical challenges associated with such type of deployments and designs as discussed in following section.

\subsection{CHALLENGES ASSOCIATED WITH DEPLOYMENT OF UAVS AS RELAYS}

This section provides a brief overview of various associated challenges in the deployment of UAVs as RSs. It also discusses potential research directions.
Based on literature review, major technical challenges in UAV deployment as RS are:

\section{Deployment of UAVs as Relay Nodes}

Optimization in UAV deployment in 3D space is one of the main challenging design considerations. Better control over trajectory, localization, and navigation in the presence of various physical factors like flight path, throughput, flight time and energy limitations which result in channel quality variations is a challenging task. The performance of multiple antenna UAVs assisted communication with optimized trajectory control has been studied in [21]. A study on ad-hoc connectivity issues of UAVs considering user location information is available with UAVs has been presented in [22]. The channel characteristics of the UAV-GBS link are a function of UAV height, flight, inter-cell interference, co-channel interference, and energy constraints are even more challenging to model in case of multiple deployments. Since height and mobility of UAVs can easily be varied, deployment of UAVs has been discussed in [23] taking advantage of freedom in deployment. Authors in [24] have derived an expression for optimal altitude, based on average path loss characteristics of a UAV to have better coverage. This research work also discusses energy characteristics, shadowing effect and coverage radius probability. In [25], UAV deployment optimization work on bit error rate is carried out considering UAV as a relay between GBS and EU to enhance connectivity. Research results show that in low altitude platforms (LAP) installed with higher beam-width directional antennas, the requirement of number of UAVs is comparatively less at reduced intercellular interference.

\section{Link Overloading}

UAV as RS has limited capacities and can communicate to limited users, unlike GBS. If it accommodates more links, access link (the link between EU to BSC or to UAV) capacity will be limited by relay link (the link between UAV to UAV and UAV to GBS) resulting in delay. Such type of backhaul queuing delay is not acceptable in time-bound applications and needs better management. For instance, authors in [26] have developed a demand for the aware mechanism which is aware of the resource demand and presents a solution to the data transmission problem in UAV based relay network in the presence of a trade-off among demand reward and power consumption.

\section{Path Selection}

UAV as RS in a cellular network may have one direct path to GBS along with multiple paths using other RSs so UAV has to choose one path based on path cost. This path cost calculation needs efficient algorithms to support link efficiency. For example, WiMAX supports 11 burst profiles for various user types. Similarity Radio 
Resource Utilization Index (RRUI) and LASER are efficient path selection algorithms. Work presented in [27] is to take advantage of UAVs mobility in relay applications using an efficient routing algorithm in an unstable and dynamically changing environment. Research work in [28] presents a mechanism of guide and control in three levels for cooperative UAVs in surveillance applications under known static threats like antiaircraft guns, missile sites, towers, poles, and buildings. In this work, in the highest level, the Lyapunov vector field is utilized based on cost functions like certainty, waypoints, position and crash probability to determine waypoints for UAV while in level 2, A* algorithms guide UAV. Authors in [29] have proposed an efficient cooperative path planning algorithm based on ant colony optimization. A number of control points using Gaussian distribution are represented on a curve and then their number, path length, turning angle, pitch rate, and coverage area are optimized.

\section{Ground-to-Air and Air-to-Air LOS Channel Modeling}

The medium between the transmitter and receiver affects the signal. The air to ground (A2G) channel characteristics which are susceptible to blockage are different from air to air (A2A) channel which are dominantly LOS and for the accurate deployment of UAVs efficient channel modeling is needed. The ideal model should consider randomness on a link so in most of the literature review researchers have dealt LOS and NLOS separately considering the altitude, location, and obstacles in the path. Authors in [30] have modeled A2G path loss for LAPs in an urban environment using a statistical propagation model by deriving a closed-loop formula for probability prediction. The authors in [31] have derived expressions for occurrence probabilities and have discussed such a model for high altitude G2A communication in urban areas considering elevation angles, LOS and NLOS links. The density of building and obstacles and other environmental factors should also be considered in using Rayleigh Distribution as defined by ITU-R [32]. Authors in [33] carrying out an experiment at $915 \mathrm{MHz}$ mounted two antennas on UAV flying at $200 \mathrm{~m}$ above ground to receive signal at 8 antenna locations on the ground. Their analysis shows that near field effects arise in such deployment and high capacity communication can be provided by UAVs using proper signaling strategies.

\section{Performance Analysis of Duplexed Relay Network of UAVs}

Contrary to terrestrial wireless networks, another challenge in performance analysis of dynamic UAV assisted wireless communication is to check the role of each design parameter such as quality of service, delay, coverage, throughput, and reliability. Authors in [34] investigated the performance of drone relays in G2A wireless communication by deriving expressions for SNR distribution and the capacity of links. Authors in [35] analyzed the outage probability of OFDM based UAV relay systems. The research work derives expressions for outage probabilities for UAV assisted relay network considering loop interferences and narrowband interferences for a full duplexed OFDM cognitive DF scheme. Simulation results of the research work show that the proposed network performs better at low values of SNR compared to half duplexed mode.

\section{Network Planning}

Cellular network planning using UAVs as relays should be carried out to address both GBS associated issues like positioning, antenna selection, frequency usage, cell allotment, and traffic, amount of signaling overhead and management of interferences along with UAV associated issues like user allocation, bandwidth, dynamics, mobility, energy limitations, and interferences. Authors in [36] have investigated the optimal assignment scheme for UAVs assisted relayed wireless networks. Problems of cell association to minimize delay is studied in [37] considering four UAVS and LAPs to serve an area of four square kilometers using two GBSs under grid-based deployment. The research work uses Gaussian distribution and standard deviation for user spatial distribution. Compared to SNR based association, these schemes reduce delay. Using optimized power in meeting minimum data rate requirements in UAVs deployment has been discussed in [38].

\section{Resource Management}

UAVs assisted wireless relay networks need efficient resource planning due to challenges like limited onboard energy, bandwidth, flight duration, mobility dynamics, processing, traffic patterns, mission nature and different types of interferences. The efficient UAV based wireless communication system design should consider all above-indicated factors. The authors in [39] have the investigated energy efficiency performance of a cache-assisted information transmission network considering multi-layer caching with wireless backhaul. They have also studied energy consumption in backhaul and access links. Authors in [40] has studied optimization in resource allocation for an energy harvesting flying drone to enhance latency and flight duration by developing a routing protocol called delay aware adaptive multi-hop.

\subsection{OPEN ISSUES AND FUTURE RESEARCH OPPERTUNITIES}

On discussing various research directions and challenges in UAV deployment as a relay in wireless communication, we can outline various research problems and associated research opportunities as: 


\section{Channel Modeling}

Usually, the received signal is not only faded out but also is noisy and to retrieve the original message signal from it, better quality modulation/demodulation techniques are required. Since A2G channels in UAVs assisted relay network differ in nature from the cellular mobile communication access channels, so they should be characterized accordingly. In channel modeling of $A 2 G$ and $A 2 A$ communication links of UAVs assisted wireless communication, real-time values of path loss and fading due to Doppler's effect on UAVs mobility along with other physical processes which can impact signal should be modeled efficiently. Similarly common channel interference increases by an increasing number of relay antennas which should be dealt with efficiently.

In wireless communications, physical layer modeling, concepts of channel state information (CSI) are essential to investigate the performance of wireless networks. If CSI is of good quality, lower order modulation is sufficient while for bad CSI, higher-order modulation is used. For establishing transmit beamforming or transmit precoding, accurate CSI is required. Scheduling operations are also based on user CSI. But if CSI varies due to Doppler's Effect introduced by user mobility, the above-mentioned issues become even more challenging in maintaining reliable data transmission. To meet such challenges due to limited RF spectrum and time-varying channels, solutions are being searched for increased spectral efficiency so that ideal DL and UL data rates are achieved. Solutions to achieve transmit energy efficiency to maintain the quality of communication by working on efficient communication algorithms. Similarly, solutions for link reliability are being searched to ensure that information from the transmitter to the receiver arrives with minimum error and minimum delay. Popular techniques are FEC which uses parity bits and MIMO which uses multiple antennas. However, tradeoffs exist here as parity bits increase processing and reduce data rates while MIMO antennas increase signal processing, HW complexity, and cost. Other solutions may be using time diversity, frequency diversity, and spatial diversity.

The research work in [41] proposes statistical models based on measurements of path loss and corresponding 3GPP territorial channel models considering correction factors. Research work in [42] studies impact of co-channel interference and channel estimation error on the performance of cellular full-duplex relay network. Expressions for outage probability under Nakagami-m fading are derived and are upgraded for high SNR for three optimization problems like optimal power allocation, optimal relay location with fixed power allocation and jointly optimal power allocation and relay location. Simulation results show that interference at the relay is negligible if we allocate sufficient power to the GBS and EUs. Techniques like ray tracing, machine learning, time-variant mobility models and long- distance path loss models may be developed and tested to characterize the physical parameters.

\section{UAV Deployment and Trajectory Optimization}

The key issues in the deployment of UAVs in the presence of terrestrial cellular networks are interference, energy and bandwidth allocation, cell association and geographical awareness. Similarly, another challenge is to optimize the deployment of UAVs which act as EU in cellular networks where handover, mobility, and interference are major issues. The problems in trajectory optimization like managing throughput, energy consumption, scheduling to achieve minimum delay, reliability and flight time need efficient solutions. Using optimization theory new solutions need to be investigated in the presence of existing ground terrestrial cellular network interferences. Literature reports that at higher altitudes, the field view, and coverage increases but currently available sensors pose accuracy issues.

The work carried out in [43] has studied the optimal transmit power and trajectory algorithm of UAVs in serving multiple ground stations simultaneously. Authors in [44] tell that trajectories of UAVs have a major impact on communication delay especially UAVs with circular trajectory serving a large number of users. Their proposed algorithm to handle this delay is based on queve occupancy and moving centers of UAVs trajectories based on link traffic. A framework of deployment of massive UAV EUs having dynamic handover methods, good channel models, better scheduling algorithms and QOS is needed.

\section{Performance Analysis and QoS}

Characterization of coverage probability, spectral efficiency, and capacity of UAVs assisted wireless communication systems should be carried out to analyze the performance. The issues like heterogeneity, mobility, spatial and temporal correlations need to be optimized. Similarity role of the trajectory in determining the performance of UAVs assisted relay network needs investigation in terms of throughput, energy efficiency, scheduling, and latency.

Authors in [45] studied problems in adopting 802.11 wireless LAN to airborne UAVs communication. The research work carried out on spectral efficiency in [46] proposes a physical layer network coding based bidirectional DF relay network based on spatial modulated full-duplex nodes. Two bits are exchanged in the one-time slot among nodes. The most significant bit is for transmit antenna selection and the least significant bit is transmitted by the selected antenna. The self-interference is modeled as Gaussian with variance based on thermal noise power. For the proposed mechanism, outage probability expressions for an end to end upper and lower bound are derived in Rayleigh fading environment. Test results show that outage performance is better compared to conventional full-duplex relay network while there is 
scope to work on extending multiple antennas. Expressions for the probability of coverage and spectral efficiency may be derived and analyzed for such type of aerial networks.

Energy efficiency, scalability, robustness, and reliability are the performance requirements from such networks which are measured in terms of performance parameters like Bit Error Rate (BER), bandwidth, coverage probability, fading, delay, link probability, packet loss, path loss and scheduling.

\section{Network Planning}

Analysis of signaling overhead, backhaul awareness and efficient cell associations for optimal transport of information are major challenges of UAV assisted relayed wireless networks. Routing allows the network to scale, to control delay, to take care of mobility, and to ensure reliability. A multi UAVs task planning algorithm is proposed in [47] for flying ad-hoc networks to visit all target points in minimum time duration. The authors in [48] have described an architecture for path planning among interconnected drones with onboard sensors, embedded processing, coordination and communication capabilities. The analysis results show strong dependency among design blocks, efficient evaluation methods, a good level of autonomy and low level of user interaction.

Development of suitable routing protocols in UAV assisted relay networks considering their dynamic 2D and $3 \mathrm{D}$ mobility in terms of node addition and removal, bandwidth and energy constraints and robustness to intermittent links is a challenging job [49]. UAV assisted relay networks require frequent handovers due to their mobility which leads to latency and packet loss, degrading quality of service and needs efficient technology to be evaluated. Other issues like determining the optimal number of UAVs to provide full coverage, frequency planning for GBS and RSs and EUs on-demand basis, network resource allocation to accommodate MRS from terrestrial cellular networks and mobility of UAVs should be studied.

\section{Mobility Issues}

Adaptive communications need to be designed with UAV mobility control to improve performance. In case UAV provides good channel conditions to ground users, it may vary its speed or topology to provide better connectivity to MUs. The requirements from mobility control are energy efficiency and maximum data rate [50]. However, due to mobility, interferences from neighboring cells are challenges to overcome. Better interference management techniques specific to UAV assisted cellular networks are needed. The mobility of nodes in such FANETs needs a proper mobility model to be followed to achieve the target objectives. These mobility models are expected at a higher autonomy level compared to other vehicular mobility models.

\section{Mission Dependent Resource Management and Synchronization}

Since UAVs as relay nodes can be deployed in a wide range of applications, issues like command and control, data communication can be handled efficiently by using a centralized resource allocation techniques. Work carried out in [51] provides an optimized strategy for energy harvesting in flying access points from GBS in D2D communication using power split relaying protocol. The experimental work carried out in [52] extends the range of battery-free RFIDs using drone relays. The prototype uses a fullduplex relay to integrate the battery-free network with RFIDs preserving phase and timing characteristics of forwarded packets. Results of research evaluate that communication is enabled at over $50 \mathrm{~m}$ through relay localization algorithm having a median accuracy of $19 \mathrm{~cm}$.

The application domains demand for network time synchronization and proper time stamping for the network traffic. The selected networking technology, software, and hardware are also application-specific. The data type may be either coordination or sensed while frequency depends on network planning. Other parameters like traffic type, throughput, delay, QoS and QoE need to be planned for such networks. To deal with monitoring overhead which is a network performance degrading factor, authors in [53] have presented an intrusion/attacker detecting system for UAVs assisted networks to detect and eject tracker efficiently. The work considers the tradeoff among detection rate and monitoring overhead of networked UAVs to make a decision on tracker ejection. The Bayesian game model is proposed and tested to detect attacks with low overhead giving reliable detection.

With an aim to reduce packet collision and packet loss, research work in [54] proposes a novel efficient data acquisition framework in UAVs assisted wireless network based on reduced transmission distance among nodes. In this work, sensor nodes and UAVs redundant data transmission links are suppressed based on a priority based frame selection scheme to increase system throughput. Different transmission priorities are assigned to different UAVs based on their location/importance in different frames and to optimize power consumption, a framework based on novel routing protocol is proposed. Authors in [55] propose and implement, an adaptive MAC framework for UAVs to switch multiple MAC protocols like CSMA and TDMA based on requirements using Raspberry Pi and MDS Radio. The experimental results show that compared to a single MAC protocol, the proposed framework can improve the desired performance.

\section{Outlook}

FANETs are expected to facilitate a lot of near-future applications in civil and military domains. Reliable communication and efficient networking are essential 
to the design requirement. This survey reports essential characteristics and requirements from a communication viewpoint. The study of the abovementioned parameters of UAV operations is key design challenges. The issue of energy efficiency can be resolved through efficient transmission power control and load/node management using efficient cross-layer protocols. Development of a framework for efficient and dynamic resource management for energy, bandwidth, traffic, flight duration, frequency, interference management, transmit power, UAV count and their trajectories in UAV assisted relay network, are needed.

\subsection{CONCLUSION}

If relayed UAVs are controlled by GBS this leads to research issues of formulating and solving challenges of centralized optimization. Machine learning can enable UAVs systems to improve performance by learning automatically from the environment and past experiences. Associated problems may be formulated at the cloud level and be optimized using advanced level mathematical transport theory. However, to solve identified challenges like handover, resource allocation and flight time optimization, even more, advanced optimization techniques based on statistical theory, probability theory, and stochastic geometry may be needed. Theories like optimization, machine learning, communication, stochastic geometry, and game theory based on distributed decision making may serve as the foundation mathematical tools to deal with problems of UAVs deployment, performance analysis, network and resource planning, trajectory control and optimal coordination. After identifying research directions and associated opportunities, we can focus on features of a robust and efficient analytical a framework to mitigate design, analysis, and optimization of UAVs based relay networks. This area is highly interdisciplinary as it requires a sound understanding of mathematical, communication, and optimization theories. Technical standards in UAVs assisted wireless communication is also being developed like IEEE $802.11 \mathrm{~g}$ for wireless ad-hoc networks, World Radio Conference 2012 agreement on 5030-5061 MHz band for navigation of unmanned aircraft including control and non-payload communication links and CSMA/CA native MAC protocol of WiFi for video streaming with relays.

UAVs based mesh and relay ad-hoc wireless networking is an evolving technology for military and civilian applications. Features of UAVs like dynamics, deployment, trajectory control, power limitations, bandwidth limitations and associated features of the physical layer, data link layer, and network layer are being researched. The scope of this review paper was to provide an overview of UAVs assisted networking by reviewing important literature that discusses issues and opportunities in using UAVs as relay nodes. To present the available information in these research areas, various issues like routing, handover, and energy efficiency have been discussed. Potential applications and examples of UAVs in wireless networking are presented along with main research directions, research challenges, and possible solutions to achieve a quality of service and energy efficiency in such networks. Finally, the features of a robust and efficient analytical framework are discussed. This paper provides a roadmap for further research in identified research directions and we expect that this review of existing knowledge may lead us to do further research in these important areas with open research issues. Also, we may find answers to questions like whether UAVs are the basis for next-generation cellular networks or only for hotspots and disaster coverage.

\section{Acknowledgement}

This research is partially supported by UTM TDR Grant: Q.J130000.3551.06G96. The authors are grateful and would like to thank the School of Electrical Engineering, Faculty of Engineering, Universiti Teknologi Malaysia (UTM) for the facilities provided and those who directly or indirectly supported this research.

\section{References}

[1] US Department of Transportation. Sep. 2013. Unmanned Aircraft System (UAS) Service Demand 2015-2035: Literature Review \& Projections of Future Usage. Tech. Rep. v.0.1, DOT-VNTSC-DoD-13-01.

[2] A. Merwaday and I. Guvenc. 2015. UAV Assisted Heterogeneous Networks for Public Safety Communications. 2015 IEEE Wireless Communications and Networking Conference Workshops (WCNCW). 329-334.

[3] I. Bor-Yaliniz and H. Yanikomeroglu. 2016 The New Frontier in Ran Heterogeneity: Multi-tier Drone-cells. IEEE Communications Magazine. 54(1 1): 48-55.

[4] Mohammad Mozaffari, Walid Saad, Mehdi Bennis, Young-Han Nam, and M'erouane Debbah2. Mar 2018. A Tutorial on UAVs for Wireless Networks: Applications, Challenges, and Open Problems. arXiv:1803.00680v1 [cs.IT].

[5] G. Kramer, M. Gastpar and P. Gupta. 2005. Cooperative Strategies and Capacity Theorems for Relay Networks. IEEE Transactions on Information Theory. 51(9): 30373063. Doi: 10.1109/TIT.2005.853304.

[6] Facebook. Connecting the World from the Sky. Facebook, Technical Report.

[7] K. P. Hui, D. Phillips and A. Kekirigoda. 2017. Beyond Lineof-Sight Range Extension with OPAL using Autonomous Unmanned Aerial Vehicles. MILCOM 2017 - 2017 IEEE Military Communications Conference (MILCOM), Baltimore, MD, 2017. 279-284.

[8] J. Xie, C. Dong, A. Li, H. Wang and W. Wang. 2017. Optimal Deployment Density for Maximum Coverage of Drone Small Cells. 2017 IEEE 86th Vehicular Technology Conference (VTC-Fall), Toronto, ON. 1-6. 
[9] A. S. Cacciapuoti, M. Caleffi, L. Paura and M. A. Rahman. 2014. Link Quality Estimators for Multi-hop Mesh Network. 2014 Euro Med Telco Conference (EMTC), Naples. 1-6.

[10] C. H. Lin, R. H. Hwang, J. J. Wu, J. F. Lee and Y. D. Lin. 2015. Integration of Spatial Reuse and Allocation for Downlink Broadcast in LTE-Advanced and WiMAX Relay Networks. IEEE Transactions on Vehicular Technology. 64(1 1): 5246-5256.

[11] W. Xiuping, Y. Feng and Z. Tian. 2018. The DF-AF Selection Relay Transmission Based on Energy Harvesting. 2018 10th International Conference on Measuring Technology and Mechatronics Automation (ICMTMA), Changsha, China. 174-177.

[12] Y. Tao, B. Li, C. Zhao, Y. Zhang and F. XU. 2017. Localization-assisted UAV Multiple Access for Future Dense Cellular Networks. 2017 17th International Symposium on Communications and Information Technologies (ISCIT), Cairns, QLD. 1-5.

[13] J. Chen, O. Esrafilian, D. Gesbert and U. Mitra. 2017. Efficient Algorithms for Air-to-Ground Channel Reconstruction in UAV-Aided Communications. 2017 IEEE Globecom Workshops (GC Wkshps), Singapore. 16.

[14] C. A. Rokhmana and R. Andaru. 2016. Utilizing UAVBased Mapping in Post Disaster Volcano Eruption. 2016 6th International Annual Engineering Seminar (InAES), Yogyakarta. 202-205.

[15] H. Kim, L. Mokdad and J. Ben-Othman. 2018. Designing UAV Surveillance Frameworks for Smart City and Extensive Ocean with Differential Perspectives. IEEE Communications Magazine. 56(4): 98-104.

[16] Y. Zhou, N. Cheng, N. Lu and X. S. Shen. 2015. Multi-UAVAided Networks: Aerial-Ground Cooperative Vehicular Networking Architecture. IEEE Vehicular Technology Magazine. 10(4): 36-44.

[17] S. Song, Y. Liu, Q. Song and L. Guo. 2017. Relay Selection and Link Scheduling in Cooperative Free-space Optical Backhauling of 5G Small Cells. 2017 IEEE/CIC International Conference on Communications in China (ICCC), Qingdao. 1-6.

[18] T. M. Hoang, N. T. Tan, N. B. Cao and L. T. Dung. 2017. Outage Probability of MIMO Relaying Full-duplex System with Wireless Information and Power Transfer. 2017 Conference on Information and Communication Technology (CICT), Gwalior, India. 1-6

[19] Tran, T. X., Le, D. V., Yue, G., \& Pompili, D. 2018. Cooperative Hierarchical Caching and Request Scheduling in a Cloud Radio Access Network. IEEE Transactions on Mobile Computing. 17(12): 2729-2743. [8323203]. https://doi.org/10.1109/TMC.2018.2818723.

[20] B. Olivieri and M. Endler. 2017. An Algorithm for Aerial Data Collection from Wireless Sensors Networks by Groups of UAVs. 2017 IEEE/RSJ International Conference on Intelligent Robots and Systems (IROS), Vancouver, BC. 967-972.

[21] F. Jiang and A. L. Swindlehurst. 2012. Optimization of UAV Heading for the Ground-to-Air Uplink. IEEE Journal on Selected Areas in Communications. 30(5): 993-1005.

[22] Z. Han, A. L. Swindlehurst, and K. Liu. 2009. Optimization of MANET Connectivity via Smart Deployment/Movement of Unmanned Air Vehicles. IEEE Transactions on Vehicular Technology. 58(7): 3533-3546.

[23] R. Yaliniz, A. El-Keyi, and H. Yanikomeroglu. 2016. Efficient 3-D Placement of an Aerial Base Station in Next Generation Cellular Networks. Proc. of IEEE International Conference on Communications (ICC). Kuala Lumpur, Malaysia.
[24] A. Hourani, K. Sithamparanathan, and S. Lardner. 2014 Optimal LAP Altitude for Maximum Coverage. IEEE Wireless Communication Letters. 3(6): 569-572.

[25] P. Zhan, K. Yu, and A. L. Swindlehurst. 2006. Wireless Relay Communications Using an Unmanned Aerial Vehicle. Proc. IEEE 7th Workshop on Signal Processing Advances in Wireless Communications, Cannes, France, July.

[26] T. Yang, F. Song, X. Chen, Y. Zhang and K. Yao. 2017. Demand-aware Backhaul Allocation in UAV Networks: A Stackelberg Optimization Approach. 2017 3rd IEEE International Conference on Computer and Communications (ICCC), Chengdu.

[27] P. Zhang, Q. Zhang, M. Jiang and Z. Feng. 2017. Cube Based Space Region Partition Routing Algorithm in UAV Networks. 23rd Asia-Pacific Conference on Communications (APCC), Perth, WA, 2017. 1-6.

[28] S. Lim and H. Bang. 2009. Waypoint Guidance of Cooperative UAVs for Intelligence, Surveillance, and Reconnaissance. 2009 IEEE International Conference on Control and Automation, Christchurch. 291-296.

[29] C. T. Cheng, K. Fallahi, H. Leung and C. K. Tse. 2009. Cooperative Path Planner for UAVs using ACO Algorithm with Gaussian Distribution Functions. 2009 IEEE International Symposium on Circuits and Systems, Taipei. 173-176.

[30] A. Al-Hourani, S. Kandeepan and S. Lardner. 2014. Optimal LAP Altitude for Maximum Coverage. IEEE Wireless Communications Letters. 3(6): 569-572.

[31] J. Holis and P. Pechac. 2008. Elevation Dependent Shadowing Model for Mobile Communications via High Altitude Platforms in Built-up Areas. IEEE Transactions on Antennas and Propagation. 56(4): 1078-1084.

[32] ITU-R. 2003. Propagation Data and Prediction Methods for the Design of Terrestrial Broadband Millimetric Radio Access Systems. Series, Radiowave Propagation. 14102.

[33] T. J. Willink, C. C. Squires, G. W. K. Colman and M. T. Muccio. 2016. Measurement and Characterization of Low-Altitude Air-to-Ground MIMO Channels. IEEE Transactions on Vehicular Technology. 65(4): 2637-2648.

[34] P. Zhan, K. Yu, and A. L. Swindlehurst. 2011. Wireless Relay Communications with Unmanned Aerial Vehicles: Performance and Optimization. IEEE Transactions on Aerospace and Electronic Systems. 47(3): 2068-2085.

[35] S. Rajkumar and S. J. Thiruvengadam. 2014. Outage Analysis of OFDM based Full Duplex Cognitive DF Relay Network in the Presence of Narrowband Interference. 2014 IEEE National Conference on Communication, Signal Processing and Networking (NCCSN), Palakkad.

[36] V. Sharma, M. Bennis, and R. Kumar. 2016. UAV-assisted Heterogeneous Networks for Capacity Enhancement. IEEE Communications Letters. 20(6): 1207-1210.

[37] M. Mozaffari, W. Saad, M. Bennis, and M. Debbah. 2017 Optimal Transport Theory for Cell Association in UAVEnabled Cellular Networks. IEEE Communications Letters. 21 (9): 2053-2056.

[38] M. Mozaffari, W. Saad, M. Bennis, and M. Debbah. 2016. Optimal Transport Theory for Power-efficient Deployment of Unmanned Aerial Vehicles. Proc. Of IEEE International Conference on Communications (ICC), May.

[39] T. X. Vu, S. Chatzinotas, B. Ottersten and T. Q. Duong. 2018. Energy Minimization for Cache-Assisted Content DeliveryNetworks with Wireless Backhaul. IEEE Wireless Communications Letters. 7(3): 332-335. Doi: 10.1109/LWC.2017.2776924.

[40] K. Papithasri and M. Babu. 2016. Efficient Multihop Dual Data Upload Clustering Based Mobile Data Collection in Wireless Sensor Network. 2016 3rd International 
Conference on Advanced Computing and Communication Systems (ICACCS), Coimbatore. 1-6

[41] K. Wang et al. 2017. Path Loss Measurement and Modeling for Low-altitude UAV Access Channels. 2017 IEEE 86th Vehicular Technology Conference (VTC-Fall), Toronto, ON, 2017. 1-5.

[42] M. K. Shukla, S. Yadav and N. Purohit. 2018. Cellular Multiuser Two-Way Relay Network With Cochannel Interference and Channel Estimation Error: Performance Analysis and Optimization. IEEE Transactions on Vehicular Technology. 67(4): 3431-3446.

[43] J. XU, Y. Zeng and R. Zhang. 2017. UAV-enabled Multiuser Wireless Power Transfer: Trajectory Design and Energy Optimization. 2017 23rd Asia-Pacific Conference on Communications (APCC), Perth, WA. 1-6.

[44] Z. M. Fadlullah, D. Takaishi, H. Nishiyama, N. Kato and R. Miura. 2016. A Dynamic Trajectory Control Algorithm for Improving the Communication Throughput and Delay in UAV-aided Networks. IEEE Network. 30(1): 100-105.

[45] D. Lee, J. Lim and H. Baek. 2018. An Airborne Communication Relay Scheme for IEEE 802.11 WLAN Based Network. 2018 International Conference on Information Networking (ICOIN), Chiang Mai, Thailand. 426-431.

[46] R. Rajesh, P. G. S. Velmurugan, S. J. Thiruvengadam and P. S. Mallick. 2017. Outage Performance of Physical Layer Network Coding Based Spatially Modulated Full Duplex Bidirectional Relay Network. 2017 International Conference on Wireless Communications, Signal Processing and Networking (WiSPNET), Chennai. 19461950.

[47] I. Bekmezci, M. Ermis and S. Kaplan. 2014. Connected Multi UAV Task Planning for Flying Ad Hoc Networks. 2014 IEEE International Black Sea Conference on
Communications and Networking (BlackSeaCom), Odessa. 28-32.

[48] Yanmaz, Evsen \& Kuschnig, Robert \& Quaritsch, Markus \& Bettstetter, Christian \& Rinner, Beate. 2011. On Path Planning Strategies for Networked Unmanned Aerial Vehicles. 212-216. 10.1109/INFCOMW.2011.5928811.

[49] L. Gupta, R. Jain and G. Vaszkun. 2016. Survey of Important Issues in UAV Communication Networks. IEEE Communications Surveys \& Tutorials. 18(2): $1123-1152$.

[50] D. W. Matolak and R. Sun. 2015. Unmanned Aircraft Systems: Air-ground Channel Characterization for Future Applications. IEEE Veh. Technol. Mag. 10(2): 7985.

[51] M. Hua, C. Li, Y. Huang and L. Yang. 2017. Throughput Maximization for UAV-enabled Wireless Power Transfer in Relaying System. 2017 9th International Conference on Wireless Communications and Signal Processing (WCSP), Nanjing. 1-5.

[52] Yunfei Ma, Nicholas Selby, Fadel Adib. 2008. Drone Relays for Battery-Free Networks. ACM.

[53] H. Sedjelmaci, S. M. Senouci and N. Ansari. 2017. Intrusion Detection and Ejection Framework against Lethal Attacks in UAV-Aided Networks: A Bayesian Game-Theoretic Methodology. IEEE Transactions on Intelligent Transportation Systems. 18(5): 1143-1153.

[54] S. Say, H. Inata, J. Liu and S. Shimamoto. 2016. PriorityBased Data Gathering Framework in UAV-Assisted Wireless Sensor Networks. IEEE Sensors Journal. 16(14): 5785-5794.

[55] W. Wang, C. Dong, H. Wang and A. Jiang. 2016. Design and Implementation of Adaptive MAC Framework for UAV Ad Hoc Networks. 2016 12th International Conference on Mobile Ad-Hoc and Sensor Networks (MSN), Hefei. 195-201. 